OPEN ACCESS

Edited by:

Shuvra Dey,

Jahangirnagar University, Bangladesh

Reviewed by:

Seyed Reza Mohebbi,

Shahid Beheshti University of Medical

Sciences, Iran

Nicola Page,

National Institute of Communicable

Diseases (NICD), South Africa

${ }^{*}$ Correspondence:

Hongmei Xu

xuhongm0095@sina.com

${ }^{\dagger}$ These authors have contributed equally to this work and share first

authorship

Specialty section:

This article was submitted to Pediatric Infectious Diseases,

a section of the journal

Frontiers in Pediatrics

Received: 01 December 2021

Accepted: 09 February 2022

Published: 03 March 2022

Citation:

Tang X, Hu Y, Zhong X-N and

Xu H-M (2022) Molecular Epidemiology of Human Adenovirus, Astrovirus, and Sapovirus Among Outpatient Children With Acute Diarrhea in Chongaing, China,

2017-2019.

Front. Pediatr. 10:826600

doi: 10.3389/fped.2022.826600

\section{Molecular Epidemiology of Human Adenovirus, Astrovirus, and Sapovirus Among Outpatient Children With Acute Diarrhea in Chongqing, China, 2017-2019}

\author{
Xiang Tang ${ }^{1,2 t}$, Yue $\mathrm{Hu}^{1,2+}$, Xiaoni Zhong ${ }^{3}$ and Hongmei $\mathrm{Xu}^{1,2 *}$ \\ ${ }^{1}$ Children's Hosptital of Chongqing Medical University, Chongqing, China, ${ }^{2}$ Ministry of Education Key Laboratory of Child \\ Development and Disorders, National Clinical Research Center for Child Health and Disorders, China International Science \\ and Technology Cooperation Base of Child Development and Critical Disorders, Chongqing Key Laboratory of Child \\ Infection and Immunity, Chongqing, China, ${ }^{3}$ School of Public Health and Management, Chongqing Medical University, \\ Chongqing, China
}

Objective: To investigate the epidemiology of human adenovirus (HAdV), human astrovirus (HAstV), and sapovirus (SaV), children with acute diarrhea in Chongqing, China from 2017 to 2019 were enrolled. Improved surveillance could provide better guidance for diarrhea prevention.

Methods: Between 2017 and 2019, fecal specimens were collected from children $<14$ years of age presenting with acute diarrhea for treatment at the outpatient department of the Children's Hospital, Chongqing Medical University. Human HAdV in the fecal specimens was detected by PCR, while RT-PCR was adopted for the detection of HAstV and SaV.

Results: A total of 1,352 fecal specimens were screened in this study. The detection rate of HAdV was 4.44\% (60/1352), HAstV was 2.81\% (38/1352), and SaV was 1.04\% (14/1352). The prevalence of enteric viruses in males was not significantly different to females $(p>0.05)$. We found $96.67 \%$ (58/60) of the HAdV-positive cases, $92.11 \%$ (35/38) of the HAstV-positive cases, and 100\% (14/14) of the SaV-positive cases among the children under 4 years old. HAdV cases were identified throughout the year, while the infection of HAstV peaked from March to May every year. By contrast, SaV was detected in May, July, and from September to December. In total, 41 strains of HAdVF were identified, including F41 (39/60) and F40 (2/60). Furthermore, A31, B3, B7, C1, C2, C5, and C6 were also detected in the study. In addition, we detected two genotypes of HAstV, HAstV-1 (34/38) and HAstV-5 (4/38), and two genotypes of SaV, Gl0.1 (13/14), Gl0.2 (1/14).

Conclusion: The enteric viruses HAdV, HAstV, and SaV contribute to the overall burden of diarrhea in Chongqing, especially in children $<4$ years of age. Two genotypes were identified for HAstV (HAstV-1 and HAstV-5) and SaV (GI.1 and Gl.2) with an additional 
nine genotypes detected in HAdV cases. While the F41 HAdV strain was predominant, HAdV-A31 was also detected in $10 \%$ of cases. The study results along with continuous surveillance of enteric viruses will aid in the design and implementation of future enteric vaccines and diarrhea mitigation strategies.

Keywords: human adenovirus, astrovirus, sapovirus, acute diarrhea, genotype

\section{INTRODUCTION}

Since 1990, mortality rate of children with diarrhea has been significantly decreased because of the improvements in water, sanitation, and hygiene $(1,2)$. However, diarrhea is still the world's second leading infectious cause of death in children under 5 years old. According to a previous report, approximately 530,000 children under the age of 5 died from diarrheal diseases in 2017 (1). Therefore, monitoring diarrheal diseases is still a priority. Watery diarrhea and vomiting can cause dehydration. Delays of admission to a hospital, incorrect or imprompt treatment may increase disease severity, which may subsequently result in death. A diarrheal episode could cause serious damages to the bodies of young children, which would take a long time to restore normal nutrition. Thus, the growth and development of children would be affected (3). Acute diarrhea could be caused by many factors, among which viral infection is the most common one (4). Previous studies have identified that rotavirus (RV), norovirus ( $\mathrm{NoV})$, human adenovirus (HAdV), sapovirus $(\mathrm{SaV})$, and human astrovirus (HAstV), are major viral etiologies of diarrheal illness (2). RV remains the leading cause of severe gastroenteritis worldwide. However, the introduction of RV vaccine could provide effective protection for children (5). Therefore, other diarrheal viruses, such as HAdV, HAstV, and SaV have attracted more attention (6). Currently, routine diagnostics are performed for RV and NoV using enzyme immunoassays (EIAs), but surveillance for HAdV, HAstV, and $\mathrm{SaV}$ in China is still limited. Therefore, additional molecular screening for these viruses was implemented at a children's hospital outpatient department for three years in this study, which would be benefit in understanding of epidemiology in SaV, HAstV, and HAdV.

Human adenovirus is a non-enveloped linear double-stranded DNA virus detected in various glands, such as the tonsils and mesenteric lymph nodes. HAdV has been divided into over 100 serotypes (HAdV Working Group, updated April, $2021^{1}$ ), which can be further grouped into seven genotypes (A-G). Different genotypes may cause different symptoms. In addition to gastroenteritis, HAdV also causes acute respiratory illness, conjunctiva, hemorrhagic cystitis, hepatitis, hemorrhagic colitis, pancreatitis, nephritis, and meningoencephalitis (7). It has been proposed that both HAdV41 and HAdV40 belong to the subgroup of $\mathrm{F}$, which are known as enteric adenoviruses (EAds) $(8,9)$.

HAstV was first identified in 1975 by Appleton and Higgins during electron microscopic examination of fecal specimens, obtained from children with acute gastroenteritis. HAstV are non-enveloped single-stranded RNA (ssRNA) viruses with

${ }^{1}$ http://hadvwg.gmu.edu genome size of $6.2-7.7 \mathrm{~kb}$, which contains three open reading frames (ORFs, that are, ORF1a, ORF1b, and ORF2). ORF1a and ORF1b encode some non-structural proteins, such as RNAdependent RNA polymerase whilst ORF2 encodes the capsid protein precursor (10). Currently, HAstV strains are categorized into eight classic genotypes (HAstV-1-HAstV-8), and two novel genotypes (HAstV-MLB and HAstV-VA/HMO). The majority of childhood diarrhea is caused by the classic HAstV genotypes and there is still uncertainty about the role of HAstV-MLB and HAstV-VA/HMO in diarrheal disease (11).

Sapovirus, a genus in the Caliciviridae family alongside NoV, is increasingly recognized as an important cause of childhood diarrhea. Overall, 19 genogroups (GI-GXIX) have been described in $\mathrm{SaV}$, with genogroups GI, GII, GIV, and GV limited to humans (including GI.1-7; GII.1-8; GIV.1; and GV.1-2) (12). SaV was rarely studied compared to $\mathrm{NoV}$ in the past. Recently, it has been shown that $\mathrm{SaV}$ infection is widely distributed. $\mathrm{SaV}$ is responsible for both sporadic cases and outbreaks of acute diarrhea.

As a city located on the intersection of two rivers (Yangtze River and Jialing River), Chongqing has extreme seasonal variations displayed in temperature ("hot summer and cold winter") compared to other cities. In the present study, three diarrheal viruses in pediatric patients in Chongqing were monitored throughout the year for the early detection of new variants in the population. Therefore, the improved surveillance could provide better guidance for diarrhea prevention.

\section{SUBJECTS AND METHODS}

\section{Subjects}

In total, 1,352 fecal specimens were collected by healthcare professionals from January 1, 2017 to December 31, 2019 at the Clinical Laboratory Center of The Affiliated Children's Hospital of Chongqing Medical University. All the collected samples were assessed in the present study. The inclusion criteria for collected samples were as follows: frequent bowel movements (three or more per day); loose, explosive, and watery stools; WBC (White blood cells) < 10 HP-1 (high power field)examined by microscopic; exclusion of both mucous stools and bloody purulent stools; and less than 2 weeks of disease duration (since onset). The collected samples were stored at $-20^{\circ} \mathrm{C}$ immediately, and repeated freezing and thawing was avoided. Written consent was obtained from the parents of all pediatric children. General information and the disease conditions of the patients were recorded. This study was approved by the medical ethics committee of the Children's Hospital of Chongqing Medical University [File No.2020 ethical review (research) No.154]. 


\section{Methods}

\section{Main Reagents}

QIAamp Viral RNA Mini Kits were purchased from QIAGEN GmbH (Hilden, Germany). SuperScript III First-Strand Synthesis System reverse transcriptase was purchased from Invitrogen Inc. (Carlsbad, CA, United States). ExTaqDNA polymerase was purchased from Takara Biotechnology (Dalian) Co., Ltd. (China).

\section{Detection of HAdV, HAstV, and SaV}

Viral RNA was extracted using the QIAamp ${ }^{\circledR}$ Viral RNA Mini Kit, while reverse transcription was performed using the SuperScript ${ }^{\circledR}$ III First-Strand Synthesis System for RT-PCR. All procedures were conducted in accordance with the instructions provided in the kits. In the present study, all samples were tested for $\mathrm{AdV}, \mathrm{SaV}$, and $\mathrm{AstV}$ using reverse transcription-polymerase chain reaction (RT-PCR), or PCR. Gene region of hexon was amplified for detection of HAdV, while gene region of capsid (ORF2) was amplified for detection of HAstV. Capsid protein VP1 was amplified for detection of $\mathrm{SaV}$. The primer pairs selected to detect HAdV, HAstV and SaV were named as Ad1 and Ad2, Mon269 and Mon270, SLV5317 and SLV5749, respectively. The size of the amplicons were $482 \mathrm{bp}$ for HAdV, $449 \mathrm{bp}$ for $\mathrm{HAstV}$, and $434 \mathrm{bp}$ for $\mathrm{SaV}$. These primer sequences were designed as previously reported $(13,14)$.

The PCR reaction conditions were as follows: predenaturation at $94^{\circ} \mathrm{C}$ for $5 \mathrm{~min}$, followed by 35 cycles of $94^{\circ} \mathrm{C}$ denaturation for $30 \mathrm{~s}, 55^{\circ} \mathrm{C}$ annealing for $30 \mathrm{~s}$, and $72^{\circ} \mathrm{C}$ extension for $1 \mathrm{~min}$, followed by a final extension at $72^{\circ} \mathrm{C}$ for $7 \mathrm{~min}$, and termination at $4^{\circ} \mathrm{C}$. Agarose gel electrophoresis (1.5\%) was performed for analysis of the PCR products.

\section{Sequence Analysis}

All virus-positive (HAdV, HAstV, and SaV) samples were subjected to gel recovery and purification before sent to Invitrogen Biotechnology (Shanghai) Co., Ltd., for sequencing. The sequencing results were processed by DNAstar software. The obtained sequences were aligned with sequences retrieved from the GenBank database using BLAST searches. A phylogenetic tree was established by MEGA 7.0 software with Kimura's 2-parameter to calculate the genetic distance, while the neighbor-joining method with boot-strap was deployed.

GeneBank database was used to obtain all reference sequences. The gene sequences described in the present study have been deposited in the GenBank database under the accession number of OL681900-OL682011.

\section{Statistical Analysis}

Statistical significance of the obtained data was determined by Excel and SPSS 23.0 software. Measurement data was denoted in the form of $(x \pm s)$, while counting data was subjected to the chisquare $\left(\chi^{2}\right)$ test. Differences at level of $P<0.05$ was considered as statistically significant.

\section{RESULTS}

In this study, a total of 1,352 stool samples collected from children with acute diarrhea were enrolled from January 1, 2017 to December 31, 2019 (including 444 samples in 2017, 475 samples in 2018, and 433 samples in 2019). Amongst all the samples, stool was sampled from 802 boys and 550 girls. All of the enrolled children had been diagnosed with acute diarrhea at the Children's Hospital of Chongqing Medical University, who were followed with attendance as outpatients. The ages of the enrolled patients ranged from 10 days to 166 months, with an average of $16.34 \pm 17.83$ months.

Among these 1,352 fecal samples, $8.28 \%(112 / 1352)$ were infected with the three enteric viruses. A total of 60 cases [4.44\% (60/1352)] with HAdV were detected in the 3 years. Specifically, the HAdV-positive rate was $4.95 \%(22 / 444)$ in $2017,5.05 \%$ $(24 / 475)$ in 2018 , and $3.23 \%(14 / 433)$ in 2019 . We also identified 38 cases with HAstV $(2.81 \%, 38 / 1352)$, with a detection rate of $3.60 \%(16 / 444)$ in $2017,2.74 \%(13 / 475)$ in 2018 , and $2.08 \%$ in 2019 (9/433). In addition, 14 cases $(1.04 \%, 14 / 1352)$ were positive for $\mathrm{SaV}$, with a SaV detection rate of $1.80 \%(8 / 444)$ in $2017,0.63 \%$ $(3 / 475)$ in 2018 , and $0.69 \%(3 / 433)$ in 2019 . The detection rates of the three viruses fluctuated in each year, however, the differences were not statistically significant $\left(\chi^{2} \mathrm{HAdV}=2.185, P=0.335 ; \chi^{2}\right.$ HAstV $\left.=1.881, P=0.390 ; \chi^{2} \mathrm{SaV}=3.301, P=0.165\right)$.

Most cases occurred in children $<4$ years with $96.67 \%$ (58/60) of HAdV infection, $92.11 \%$ (35/38) of HAstV infection, and 100\% $(14 / 14)$ of $\mathrm{SaV}$ infection detected. Among all age groups, children 25-36 months showed the highest HAdV-detection rate [9.30\% $(8 / 86)]$. In contrast, children 49-60 months and $>60$ months showed the highest HAstV infection rate (4.54\%). However, only one case infected with HAstV were detected in the children 4960 months and two cases infected with HAstV were detected in the children in the $>60$ months category. Children 13-18 months had the highest rate $(2.34 \%, 6 / 256)$ of SaV detected. Taken together, the total positive rate of the three viruses gradually increased from six months to three years old, and gradually decreased after three years old. In contrast, the positive rate was relatively low in children under six months of age (Table 1).

Human adenovirus infections were identified throughout the year, accompanied with a peak infection in May $(19.05 \%)$ of 2017, two peaks in March (16.67\%) and November (14.93\%) of 2018, and one peak in July (12.50\%) of 2019. These results indicate that there was no fixed season for HAdV infection in Chongqing. HAstV were detected in all months except July and September, with a peak infection in May (14.3\%) of 2017, a peak in March (11.1\%) of 2018, a peak in March (9.30\%) of 2019, suggesting that $\mathrm{HAstV}$ was prevalent in spring in Chongqing. $\mathrm{SaV}$ was detected in May, July, September to December, with no detection in the remaining months. Notably, infections of $\mathrm{SaV}$ were mainly around May and November, suggesting that $\mathrm{SaV}$ was prevalent at the turn of autumn and winter or the turn of spring and summer in Chongqing (Figure 1).

The most common clinical symptom of the patients infected with HAdV was diarrhea $(100.0 \%, 60 / 60)$, followed by vomiting $(50 \% \%, 30 / 60)$, fever $(36.67 \%, 22 / 60)$, and cough $(45 \%, 27 / 60)$. The common clinical symptoms of HAstV-infected patients were diarrhea $(100.0 \%, 38 / 38)$, fever $(28.95 \%, 11 / 38)$, vomiting $(50 \%, 19 / 38)$ and cough $(50 \%, 19 / 38)$. In addition, the common clinical symptoms of classic SaV-infected patients were diarrhea $(100.0 \%, 14 / 14)$, fever $(7.14 \%, 1 / 14)$, vomiting $(64.29 \%, 9 / 14)$, 
TABLE 1 | Distribution of viral pathogens in 1352 children with acute gastroenteritis different age group in Chongqing.

\begin{tabular}{|c|c|c|c|c|c|c|}
\hline Age group (months) & Samples & Total $n(\%)$ & HAdV $n(\%)$ & HAstV $n(\%)$ & SaV n (\%) & Mixed infection $n(\%)$ \\
\hline $0-6$ & 312 & $9(2.88)$ & $3(0.96)$ & $6(1.92)$ & $0(0)$ & $0(0)$ \\
\hline $7-12$ & 444 & $35(7.88)$ & $21(4.73)$ & $12(2.7)$ & $2(0.45)$ & $1(0.23)^{a}$ \\
\hline $13-18$ & 256 & $26(10.16)$ & $13(5.08)$ & 7 (2.73) & $6(2.34)$ & $0(0)$ \\
\hline $19-24$ & 131 & $17(12.98)$ & $9(6.87)$ & $5(3.82)$ & $3(2.29)$ & $2(1.53)^{b}$ \\
\hline $25-36$ & 86 & $13(15.12)$ & $8(9.3)$ & $3(3.49)$ & $2(2.33)$ & $0(0)$ \\
\hline $37-48$ & 57 & 7 (12.28) & $4(7.02)$ & $2(3.51)$ & $1(1.75)$ & $0(0)$ \\
\hline $49-60$ & 22 & $1(4.55)$ & $0(0)$ & $1(4.55)$ & $0(0)$ & $0(0)$ \\
\hline$>60$ & 44 & $4(9.09)$ & $2(4.55)$ & $2(4.55)$ & $0(0)$ & $0(0)$ \\
\hline Total & 1352 & 112 (8.28) & $60(4.44)$ & $38(2.81)$ & $14(1.04)$ & $3(0.22)$ \\
\hline
\end{tabular}

n(\%) denotes number and consituent ratio. ${ }^{a}$ Tri-viral mixed infection of HAdV-F41 + HAstV-1 + GI0.2. ${ }^{b}$ Bi-viral mixed infection occurred in two cases.

and cough $(28.57 \%, 4 / 14)$. The occurrence of diarrhea and vomiting was significantly higher in SaV-positive cases (64.29\%; 9/14) compared to SaV-negative cases (47.38\%; 634/1338; $P=0.002$ ) (Table 2).

During the study period, four subgroups of HAdV were detected, including HAdV-A (6/60,10\%), HAdV-B (6/60,10\%), HAdV-C (7/60,11.67\%), and HAdV-F (41/60,68.33\%). HAdVF41 was the most common genotype [65.00\% (39/60)] among HAdV cases, followed by A31 (6/60,10\%), B3 (5/60,8.3\%), C5 (3/60,5.0\%), F40 (2/60,3.3\%), C6 (2/60,3.3\%), B7 (1/60,1.67\%), C1 $(1 / 60,1.67 \%)$, and C2 $(1 / 60,1.67 \%)$. As two causative factors of respiratory infection, $\mathrm{HAdV}-\mathrm{B}$ and $\mathrm{HAdV}-\mathrm{C}$ were associated with the respiratory tract symptoms in 7 out of 13 pediatric patients who were infected with HAdV-B or HAdV-C. Phylogenetic analysis showed two clusters of F41 strains circulating in this study. Two F40 strains were detected in 2017, and no A31 strain were detected in 2019 (Figure 2). Two different genotypes of HAstV (HAstV-1 and HAstV-5) were identified among 38 samples, while HAstV-1 (34/38,89.47\%) was the predominant genotype. Phylogenetic analysis revealed three clusters of HAstV1 strains. The major cluster (26 strains) was found to be closely related to the reference strain previously isolated from Thailand (HQ398856) in 2017, which showed 98.57-99.72\% nucleotide sequence identity. Additionally, four HAstV-5 strains were detected in 2017 (Figure 3). Two genotypes of SaV were detected, including GI0.1 (13/14,92.9\%) and GI0.2 (1/14,7.1\%), with one GI0.2 strain was detected in 2017. In addition, the cluster with 13 strains was found to be closely related to the reference strain isolated from chongqing (KF495124) in 2010 (that is 99.04-99.36\% nucleotide sequence identity) (Figure 4).

Among the 1352 specimens, there were three cases of mixed infection, which were caused by HAdV and other viruses concomitantly. Bi-viral mixed infections were also detected, which occurred in two cases, including HAdV-F41 + HAstV-1 and HAdV-F41 + GI0.1. We also detected the Tri-viral mixed infection, which occurred in one case (HAdV-F41 + HAstV$1+$ GI0.2). All the three cases displayed watery stool. However, one case showed none other clinical symptoms, one showed lowgrade fever and the other showed respiratory tract infection. The three cases of mixed infection ranged in age from 12 months to 21 months, which suggests that the mixed infection was prevalent under 2 years old.

\section{DISCUSSION}

Acute infectious diarrhea in children is mainly caused by viruses, while RV and NoV are the two most common viruses (15). HAdV, HAstV, and SaV contributed to the overall diarrheal burden in China. This study mainly monitored the infection of the above three viruses in children exhibiting acute diarrhea from 2017 to 2019 in Chongqing, and all the basic data would provide a theoretical foundation for formulating a strategy to prevent and control viral diarrhea.

In Chongqing, $4.44 \%$ of pediatric patients with acute diarrhea were diagnosed with HAdV infection during 2017 and 2019, which was close to the HAdV prevalence in Hangzhou (3.1\%, 2017-2018) (16) and Shanghai (3.47\%, 2017-2018) (17) reported in recent studies of pediatric diarrhea with $\mathrm{HAdV}$ infection in China. HAdV infections are more prevalent in pediatric patients with diarrhea in African countries such as Nigeria (19.3\%) (18) and Gabon (19.6\%) (19), suggesting a regional difference in HAdV prevalence and correlations of HAdV prevalence with economic development and hygiene conditions. In Chongqing, F41 was more frequent than F40, which was consistent with the findings reported by other studies in Chongqing and other regions in China $(15-17,20,21)$. Conversely, HAdV-40 was detected more frequently than F41 in Kolkata, India although the study period was different (2013-2014) (9). HAdV-A was also associated with diarrhea (8) and we detected six A31 strains in this study, which was the second predominant genotype followed by F41, which was similar to the results of a study in Beijing (21). This demonstrated that A31 was an important genotype causing acute diarrhea in pediatric patients. Additionally, 13 strains of HAdV-B and HAdV-C were detected in the present study. Meanwhile, Qiu et al. (22) reported that B3 was a high-risk factor for diarrhea through a comparison of the stool detection rate between pediatric patients with diarrhea and healthy children after the exclusion of the possibility of co-infection with other intestinal pathogens. Gelaw et al. (23) and Kumthip et al. (24) reported that pediatric patients with diarrhea had a higher stool detection rate of HAdV-C than that of HAdV-F, while the HAdV subgroup $\mathrm{C}$ was the most prevalent genotype in both Northwest Ethiopia and Thai. However, it remains unknown whether diarrhea is directly caused by HAdV-B or HAdV-C. A possible reason for the coincidental detection of HAdV-B or 


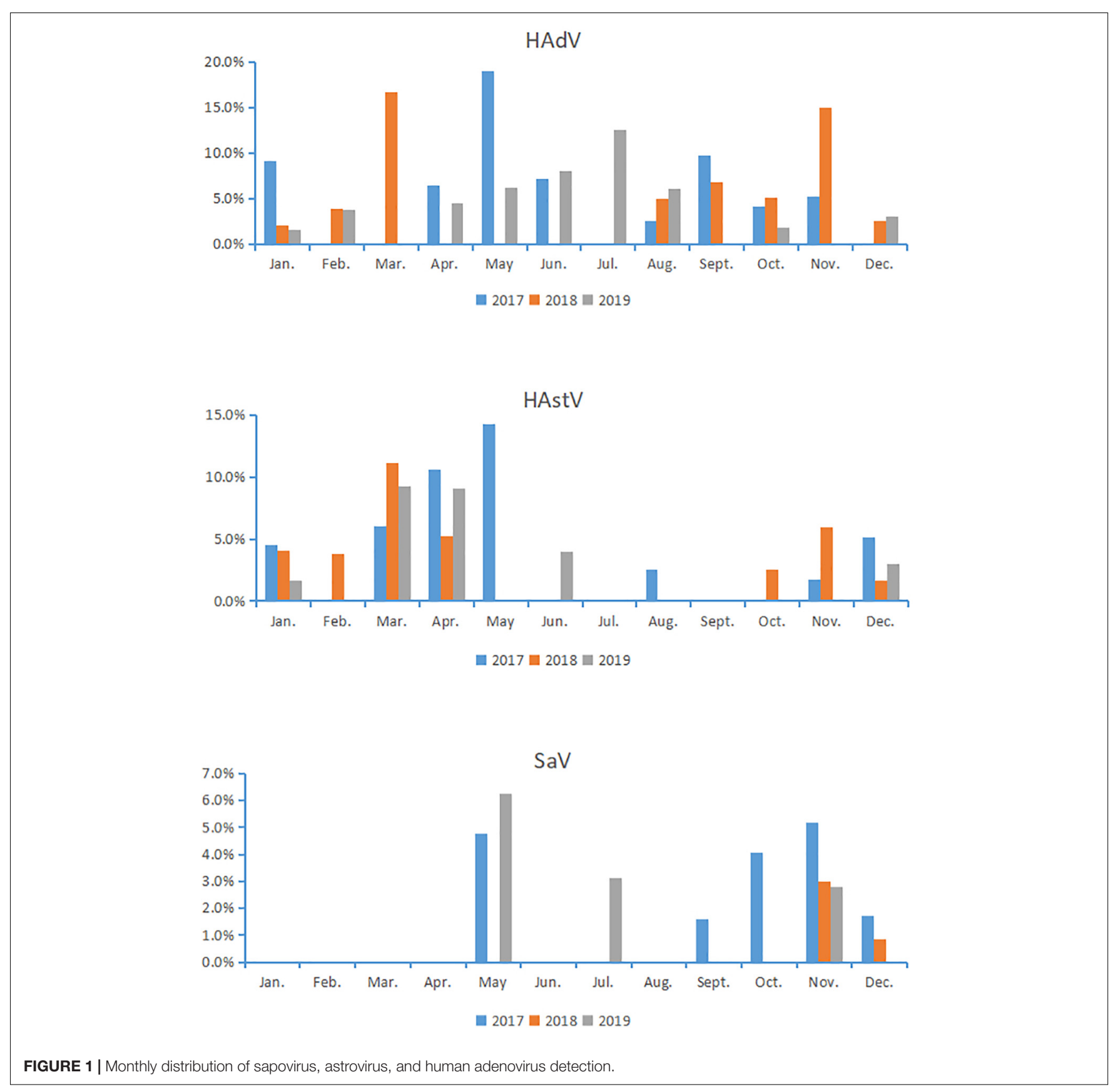

TABLE 2 | Clinical symptoms of diarrhea children infected with and without HAdV, HAstV and SaV.

\begin{tabular}{|c|c|c|c|c|c|c|}
\hline Symptoms & \multicolumn{2}{|c|}{ HAdV n (\%) } & \multicolumn{2}{|c|}{ HAstV n (\%) } & \multicolumn{2}{|c|}{ SaV n (\%) } \\
\hline diarrhea & $60(100)$ & $1292(100)$ & $38(100)$ & $1314(100)$ & $14(100)$ & 1338 (100) \\
\hline fever & $22(36.67)$ & 423 (32.74) & $11(28.95)$ & 434 (33.03) & $1(7.14)$ & $444(33.18)$ \\
\hline vomiting & $30(50.00)$ & $613(47.45)$ & $19(50.00)$ & 624 (47.49) & 9 (64.29) & $634(47.38)^{a}$ \\
\hline cough & $27(45.00)$ & 512 (39.63) & $19(50.00)$ & 520 (39.57) & $4(28.57)$ & 535 (39.99) \\
\hline
\end{tabular}

${ }^{a}$ The difference between infected and uninfected SaV group was statistically significant when the clinical symptoms of vomiting $(P=0.002)$. 


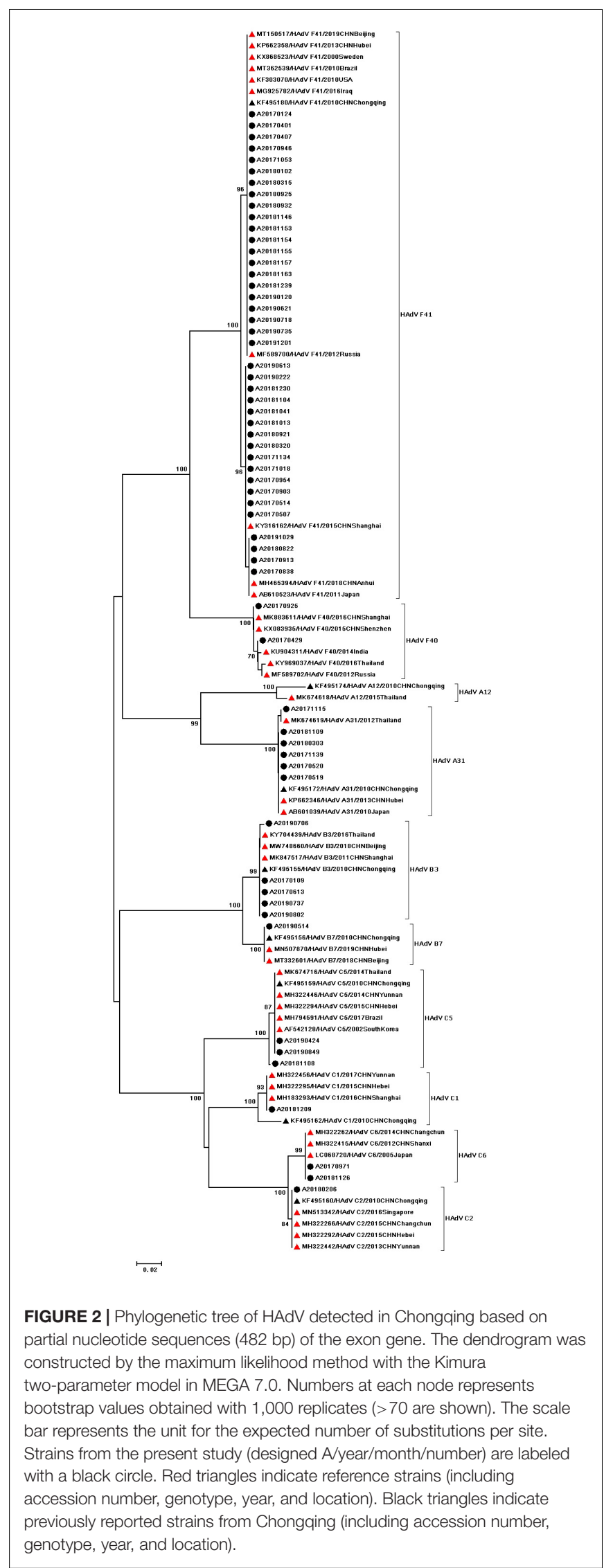

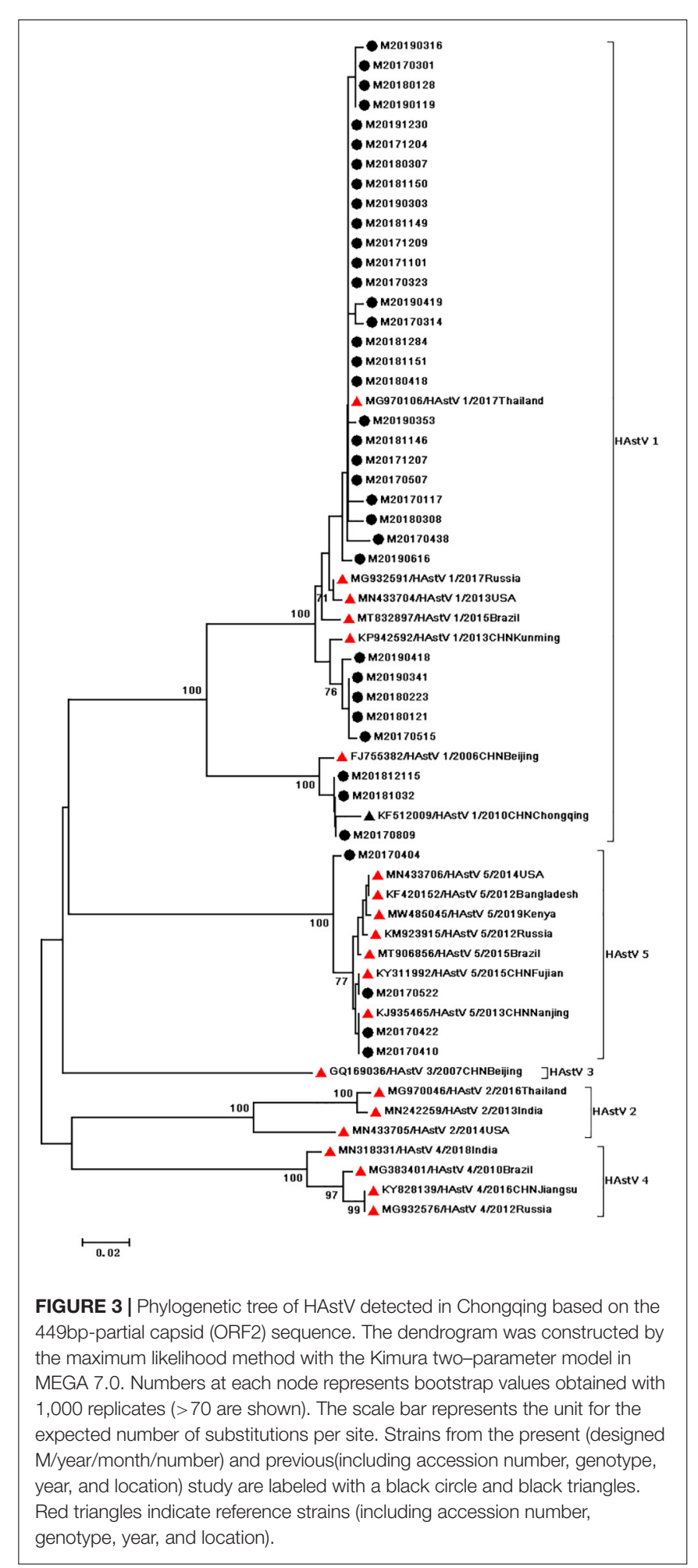

HAdV-C in stool could be the presence of an asymptomatic elsewhere in the body or shedding of the virus after a recent respiratory, ocular or urinary tract infection. Therefore, more work will be required to establish the association in future. 


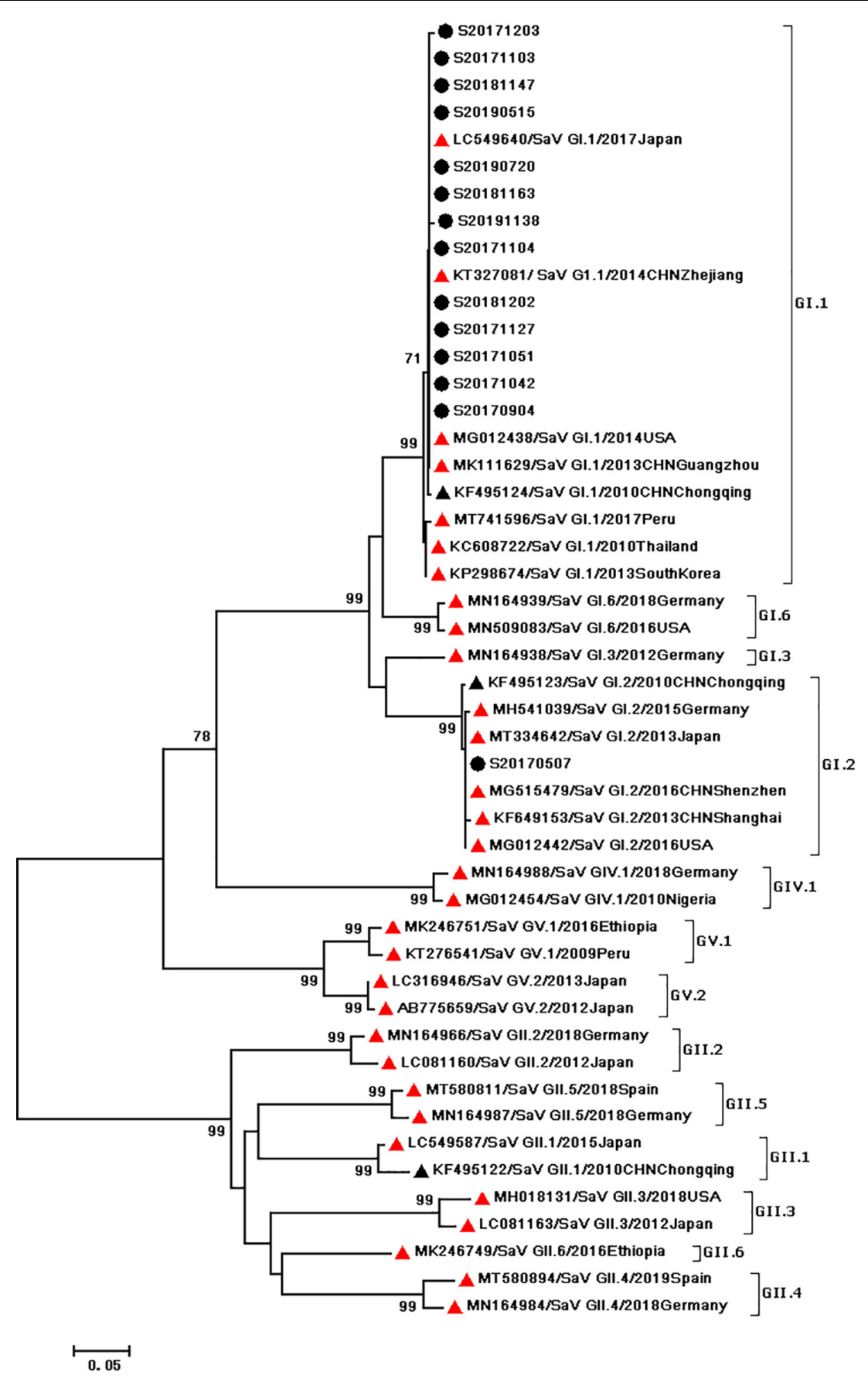

FIGURE 4 | Phylogenetic tree of SaV detected in Chongqing based on the 434bp-partial capsid sequence. The dendrogram was constructed by the maximum likelihood method with the Kimura two-parameter model in MEGA 7.0. Numbers at each node represents bootstrap values obtained with 1,000 replicates (>70 are shown). The scale bar represents the unit for the expected number of substitutions per site. Strains from the present (designed S/year/month/number) and previous(including accession number, genotype, year, and location) study are labeled with a black circle and black triangles. Red triangles indicate reference strains (including accession number, genotype, year, and location).

The present study showed that during the year from 2017 to 2019, the overall HAstV-positive rate in Chongqing was 2.81\% in pediatric patients with acute diarrhea, similar to that in Thai (2.6\%) (25). The HAstV infection rates were slightly higher in Shanghai (5.22\%) (17) and eight low-income countries (5.6\%) in Asia, Africa, and South America (e.g., Bangladesh, Tanzania, Peru) (26). In Japan, the HAstV infection rate was $16.4 \%$ (27), markedly higher than the results of this study. Mon269 and Mon270 were used for nucleotide sequence amplification in the region of ORF2 of HAstV to identify the genotype of HAstV1-8. 
Consequently, none of novel HAstV (e.g., HAstV-MLB, HAstV$\mathrm{VA} / \mathrm{HMO}$ ) were detected in this study. In the present study, two genotypes, namely HAstV-1 and HAstV-5, were detected. HAstV1 was the predominant strain in our study. In contrast, HAstV-2 was detected in Chongqing in 2014 (20). HAstV-1 has been recognized as a predominant circulating genotype worldwide, followed by HAstV-2-5 and HAstV-8, whereas HAstV-6 and HAstV-7 are rarely detected (10). Japanese researchers reported that primers SF0073 and SF0076 could simultaneously detect both classic and novel HAstVs, which could yield a higher positive rate $(16.4 \%)$. However, it is still controversial whether the novel HAstVs could result in diarrhea (11). In fact, only $4.8 \%$ of the samples were classic HAstV strains in Japan. Moreover, Japanese researchers have demonstrated that over $50 \%$ of all HAstV-MLB infections occurred in parallel with both RV and $\mathrm{NoV}$, which resulted in the difficulty in the identification of the primary pathogen (27). In this study, infection of HAstV occurred most frequently during March and May, while HAstV5 infection was not detected until April and May, indicating that HAstV infections might be epidemic in the spring. It had been reported that HAstV infection was prevalent in January, April and November to December in Chongqing in 2014 (20). Therefore, the infection of HAstV might be associated with the low environmental temperature, thus displayed a typical winter/spring seasonality. In addition, our results were also consistent with the results observed in Shanghai in 2015-2016, which also displayed a typical winter/spring seasonality (28). However, the infection peaks of HAstV varied in different years in this study, and the seasonality of HAstV infections in Chongqing needs to be further monitored.

The frequency of $\mathrm{SaV}$ is variable across geographic locations and age groups. However, $\mathrm{SaV}$ has been detected in $1-17 \%$ of diarrhea in various settings (12). Nevertheless, this study indicated that the $\mathrm{SaV}$ prevalence was not high in Chongqing, with an infection rate of $1.04 \%$, which was close to the reported rate of $0.69 \%$ in eight cities during 2012-2014 in China (29). In Chongqing, $\mathrm{SaV}$ infections occurred more frequently in May or November. Likewise, in the Netherlands, SaV was most commonly detected in the winter and spring (30). Infants aged 7-48 months were affected by the virus. In this study, vomiting was a typical clinical symptom of $\mathrm{SaV}$ infection, whereas fever was rarely observed (Table 2). Similarly, a birth cohort in Peru reported that $40 \%$ children infected with $\mathrm{SaV}$ diarrhea experienced vomiting and 10\% experienced fever (31). In surveillance studies of children with SaV diarrhea, the GI.1 genotype was common ranging in prevalence from 10 to $63 \%$, followed by GI.2 and GII.1 (12, 32). Moreover, GI genotypes were prevalant during previous epidemics in Chongqing $(15,20)$. Notably, SaV outbreaks have been reported in high-income countries, particularly in those possessing a large child population $(33,34)$. Besides, SaV outbreaks occur frequently in China $(35,36)$. Therefore, SaV outbreak surveillance deserve attention in future study.

In this study, we also detected three mixed infection cases. However, we found no significant difference between the mixed infection cases and the non-mixed infection cases in terms of the severity of the disease. We also noted that the mixed infection occurred among young infants and toddlers. Our results were in consistent with previous reports $(37,38)$.

\section{CONCLUSION}

The enteric viruses, HAdV, HAstV, and SaV, contribute to the overall burden of diarrhea in outpatient cases in Chongqing, especially in children $<4$ years of age. HAdV was found year-round while HAstV and $\mathrm{SaV}$ displayed some seasonality. Predominant circulating viral strains included HAdV-F41, HAstV-1, and SaV GI.1 with the study contributing sequence data to global molecular epidemiology of enteric viruses. Continuous surveillance of enteric viruses is recommended to aid in the design and implementation of future diarrhea mitigation strategies and enteric vaccines.

\section{LIMITATION OF THIS STUDY}

Only three enteric viruses were investigated during the study and expanded molecular testing for $\mathrm{RV}, \mathrm{NoV}$ as well as enteric bacteria and parasites would provide a holistic picture of the pathogens contributing to diarrheal disease in Chongqing. In addition, the primers used to detect HAstV strains may bias the results, detecting only classic or recombinant HAstV strains and not novel HAstV.

\section{DATA AVAILABILITY STATEMENT}

Publicly available datasets were analyzed in this study. This data can be found here: OL681900-OL682011.

\section{ETHICS STATEMENT}

The studies involving human participants were reviewed and approved by Institutional Review Board of Children's Hospital of Chongqing Medical University. Written informed consent to participate in this study was provided by the participants' legal guardian/next of kin. Written informed consent was obtained from the minor(s)' legal guardian/next of kin for the publication of any potentially identifiable images or data included in this article.

\section{AUTHOR CONTRIBUTIONS}

$\mathrm{XT}$ and YH performed the experiments, analyzed the data, and wrote the manuscript. H-MX designed the study and revised the manuscript. X-NZ coordinated the study and analyzed the data. All authors approved the final version of the manuscript.

\section{FUNDING}

This study was supported by Major project in infectious diseases, Communicable Disease Surveillance platform (2017ZX10103010-003). 


\section{REFERENCES}

1. Troeger CE, Khalil IA, Blacker BF, Biehl MH, Albertson SB, Zimsen SRM, et al. Quantifying risks and interventions that have affected the burden of diarrhoea among children younger than 5 years: an analysis of the Global Burden of Disease Study 2017. Lancet Infect Dis. (2020) 20:37-59. doi: 10.1016/S14733099(19)30401-3

2. Troeger C, Blacker BF, Khalil IA, Rao PC, Cao S, Zimsen SR, et al. Estimates of the global, regional, and national morbidity, mortality, and aetiologies of diarrhoea in 195 countries: a systematic analysis for the Global Burden of Disease Study 2016. Lancet Infect Dis. (2018) 18:1211-28. doi: 10.1016/S14733099(18)30362-1

3. Troeger C, Colombara DV, Rao PC, Khalil IA, Brown A, Brewer TG, et al. Global disability-adjusted life-year estimates of long-term health burden and undernutrition attributable to diarrhoeal diseases in children younger than 5 years. Lancet Glob Health. (2018) 6:e255-69. doi: 10.1016/S2214-109X(18) 30045-7

4. Platts-Mills JA, Liu J, Rogawski ET, Kabir F, Lertsethtakarn P, Siguas M, et al. Use of quantitative molecular diagnostic methods to assess the aetiology, burden, and clinical characteristics of diarrhoea in children in low-resource settings: a reanalysis of the MAL-ED cohort study. Lancet Glob Health. (2018) 6:e1309-18. doi: 10.1016/S2214-109X(18)30349-8

5. Troeger C, Khalil IA, Rao PC, Cao S, Blacker BF, Ahmed T, et al. RV vaccination and the global burden of rotavirus diarrhea among children younger than 5 years. JAMA Pediatr. (2018) 172:958-65. doi: 10.1001/ jamapediatrics.2018.1960

6. Chaaithanya IK, Bhattacharya D, Patil T, Ghargi KV, Kalal S, Roy S, et al. Etiology of non-rotaviral diarrhea in hospitalized children under five years of age. Indian J Pediatr. (2020) 87:571-2. doi: 10.1007/s12098-02003299-8

7. Lynch JP III, Kajon AE. Adenovirus: epidemiology, global spread of novel serotypes, and advances in treatment and prevention. Semin Respir Crit Care Med. (2016) 37:586-602. doi: 10.1055/s-0036-158 4923

8. Brown M, Grydsuk JD, Fortsas E, Petric M. Structural features unique to enteric adenoviruses. Arch Virol Suppl. (1996) 12:301-7. doi: 10.1007/978-37091-6553-9_32

9. Banerjee A, De P, Manna B, Chawla-Sarkar M. Molecular characterization of enteric adenovirus genotypes 40 and 41 identified in children with acute gastroenteritis in Kolkata, India during 2013-2014. J Med Virol. (2017) 89:60614. doi: 10.1002/jmv.24672

10. Cortez V, Meliopoulos VA, Karlsson EA, Hargest V, Johnson C, Schultz-Cherry S, et al. Astrovirus biology and pathogenesis. Annu Rev Virol. (2017) 4:327-48. doi: 10.1146/annurev-virology-101416-041742

11. Diem-Lan V, Albert B, Rosa P, Guix S. Epidemiology of classic and novel human astrovirus: gastroenteritis and beyond. Viruses. (2017) 9:33. doi: 10 . 3390/v9020033

12. Becker-Dreps S, González F, Bucardo F. Sapovirus: an emerging cause of childhood diarrhea. Curr Opin Infect Dis. (2020) 33:388-97. doi: 10.1097/ QCO.0000000000000671

13. Thongprachum A, Khamrin P, Pham NT, Takanashi S, Okitsu S, Shimizu H, et al. Multiplex RT-PCR for rapid detection of viruses commonly causing diarrhea in pediatric patients. J Med Virol. (2017) 89:818-24. doi: 10.1002/jmv. 24711

14. Shrestha SK, Shrestha J, Andreassen AK, Strand TA, Dudman S, Dembinski JL. Genetic diversity of astrovirus in children from a birth cohort in Nepal. Front Microbiol. (2021) 11:3537. doi: 10.3389/FMICB.2020.58 8707

15. Ren Z, Kong Y, Wang J, Wang Q, Huang A, Xu H, et al. Etiological study of enteric viruses and the genetic diversity of norovirus, sapovirus, adenovirus, and astrovirus in children with diarrhea in Chongqing, China. BMC Infect Dis. (2013) 13:412. doi: 10.1186/1471-2334-13-412

16. Li W, Xiang W, Li C, Xu J, Zhou D, Shang S, et al. Molecular epidemiology of rotavirus $\mathrm{A}$ and adenovirus among children with acute diarrhea in Hangzhou, China. Gut Pathog. (2020) 12:19. doi: 10.1186/s13099-020-00 359-4

17. Lu L, Zhong H, Xu M, Su L, Cao L, Jia R, et al. Molecular and epidemiological characterization of human adenovirus and classic human astrovirus in children with acute diarrhea in Shanghai, 2017-2018. BMC Infect Dis. (2021) 21:713. doi: 10.1186/S12879-021-06403-1

18. Imade PE, Eghafona NO. Viral agents of diarrhea in young children in two primary health centers in Edo State, Nigeria. Int J Microbiol. (2015) 2015:685821. doi: 10.1155/2015/685821

19. Lekana-Douki SE, Kombila-Koumavor C, Nkoghe D, Drosten C, Drexler JF, Leroy EM, et al. Molecular epidemiology of enteric viruses and genotyping of rotavirus A, adenovirus and astrovirus among children under 5 years old in Gabon. Int J Infect Dis. (2015) 34:90-5. doi: 10.1016/j.ijid.2015. 03.009

20. Wanbing C, Feng Y, Yingqin Q, Hongmei X. The prevalence of astrovirus, sapovirus, and adenovirus enteric infections in children with acute diarrhea in Chongqing in 2014. Clin Pediatr. (2016). 34:241-5. doi: 10.3969/j.issn.10003606.2016.04.001

21. Liu L, Qian Y, Zhang Y, Zhao L, Jia L, Dong H, et al. Epidemiological aspects of rotavirus and adenovirus in hospitalized children with diarrhea: a 5-year survey in Beijing. BMC Infect Dis. (2016) 16:508. doi: 10.1186/s12879-0161829-z

22. Qiu F, Shen X, Li G, Zhao L, Chen C, Duan S, et al. Adenovirus associated with acute diarrhea: a case-control study. BMC Infect Dis. (2018) 18:450. doi: 10.1186/s12879-018-3340-1

23. Gelaw A, Pietsch C, Liebert UG. Genetic diversity of human adenovirus and human astrovirus in children with acute gastroenteritis in Northwest Ethiopia. Arch Virol. (2019) 164:2985-93. doi: 10.1007/s00705-019-04 421-8

24. Kumthip K, Khamrin P, Ushijima H, Maneekarn N. Enteric and non-enteric adenoviruses associated with acute gastroenteritis in pediatric patients in Thailand, 2011 to 2017. PLoS One. (2019) 14:e0220263. doi: 10.1371/journal. pone. 0220263

25. Kumthip K, Khamrin P, Ushijima H, Maneekarn N. Molecular epidemiology of classic, MLB and VA astroviruses isolated from < 5 year-old children with gastroenteritis in Thailand, 2011-2016. Infect Genet Evol. (2018) 65:373-9. doi: 10.1016/j.meegid.2018.08.024

26. Olortegui MP, Rouhani S, Yori PP, Salas MS, Trigoso DR, Mondal D, et al. Astrovirus infection and diarrhea in 8 countries. Pediatrics. (2018) 141:e20171326. doi: 10.1542/peds.20171326

27. Robinson CM, Seto D, Jones MS, Dyer DW, Chodosh J. Molecular evolution of human species D adenoviruses. Infect Genet Evol. (2011) 11:1208-17. doi: 10.1016/j.meegid.2011.04.031

28. Wu L, Teng Z, Lin Q, Liu J, Wu H, Kuang X, et al. Epidemiology and genetic characterization of classical human astrovirus infection in Shanghai, 2015-2016. Front Microbiol. (2020) 11:570541. doi: 10.3389/fmicb.2020.57 0541

29. Zhou Y, Jin M, Kong X, Huiying L, Qing Z, Xiaoman S, et al. The analysis of sapovirus infection in children under 5 years old with diarrhea in eight provinces of China, 2012-2014. Int J Virol. (2016) 23:81-4. doi: 10.3760/cma.j. issn.1673-4092.2016.02.003

30. Heusinkveld M, Mughini-Gras L, Pijnacker R, Vennema H, Scholts R, van Huisstede-Vlaanderen KW, et al. Potential causative agents of acute gastroenteritis in households with preschool children: prevalence, risk factors, clinical relevance and household transmission. Eur J Clin Microbiol Infect Dis. (2016) 35:1691-700. doi: 10.1007/s10096-0162714-9

31. Liu X, Jahuira H, Gilman RH, Alva A, Cabrera L, Okamoto M, et al. Etiological role and repeated infections of sapovirus among children aged less than 2 years in a cohort study in a peri-urban community of Peru. J Clin Microbiol. (2016) 54:1598-604. doi: 10.1128/JCM.03133-15

32. Oka T, Wang Q, Katayama K, Saif LJ. Comprehensive review of human sapoviruses. Clin Microbiol Rev. (2015) 28:32-53. doi: 10.1128/CMR.000 11-14

33. Iritani N, Yamamoto SP, Abe N, Kubo H, Oka T, Kaida A, et al. Epidemics of GI. 2 sapovirus in gastroenteritis outbreaks during 2012- 2013 in Osaka City, Japan. J Med Virol. (2016) 88:1187-93. doi: 10.1002/jmv. 24451

34. Hassan-Ríos E, Torres P, Muñoz E, Matos C, Hall AJ, Gregoricus N, et al. Sapovirus gastroenteritis in preschool center, Puerto Rico, 2011. Emerg Infect Dis. (2013) 19:174. doi: 10.3201/eid1901.120690 
35. Wang J, Li Y, Kong X, Li H, Zhang Q, Jin M. Two gastroenteritis outbreaks caused by sapovirus in Shenzhen, China. J Med Virol. (2018) 90:1695-702. doi: 10.1002/jmv.25236

36. Yan Y, Li Y, Shi W, Kong X, Li H, Zhang Q, et al. An outbreak of gastroenteritis associated with a novel GII.8 sapovirus variant-transmitted by vomit in Shenzhen, China, 2019. BMC Infect Dis. (2020) 20:911. doi: 10.1186/s12879020-05643-x

37. Oliveira-Tozetto SD, Santiso-Bellón C, Ferrer-Chirivella JM, Navarro-Lleó N, Vila-Vicent S, Rodríguez-Díaz J, et al. Epidemiological and genetic characterization of sapovirus in patients with acute gastroenteritis in Valencia (Spain). Viruses. (2021) 13:184. doi: 10.3390/v1302 0184

38. Gupta S, Krishnan A, Sharma S, Kumar P, Aneja S, Ray P, et al. Changing pattern of prevalence, genetic diversity, and mixed infections of viruses associated with acute gastroenteritis in pediatric patients in New Delhi, India. J Med Virol. (2018) 90:469-76. doi: 10.1002/jmv.24980
Conflict of Interest: The authors declare that the research was conducted in the absence of any commercial or financial relationships that could be construed as a potential conflict of interest.

Publisher's Note: All claims expressed in this article are solely those of the authors and do not necessarily represent those of their affiliated organizations, or those of the publisher, the editors and the reviewers. Any product that may be evaluated in this article, or claim that may be made by its manufacturer, is not guaranteed or endorsed by the publisher.

Copyright (c) 2022 Tang, Hu, Zhong and Xu. This is an open-access article distributed under the terms of the Creative Commons Attribution License (CC BY). The use, distribution or reproduction in other forums is permitted, provided the original author(s) and the copyright owner(s) are credited and that the original publication in this journal is cited, in accordance with accepted academic practice. No use, distribution or reproduction is permitted which does not comply with these terms. 